\title{
Cutting delays in reversing anticoagulation after intracerebral haemorrhage: three key changes at a UK comprehensive stroke centre
}

Adrian Parry-Jones

Salford Royal NHS Foundation Trust

\begin{abstract}
Prothrombin complex concentrate (PCC) reduces the risk of early haematoma expansion after intracerebral haemorrhage in patients taking vitamin $\mathrm{K}$ antagonists $(\mathrm{VKA}-\mathrm{ICH})$, so must be given without delay. We sought to identify and remove key barriers to rapid administration of PCC at our centre. We describe a single UK comprehensive stroke centre cohort study with mixed retrospective (1/1/2008 to 1/12/2010) and prospective (1/12/2010 to 31/7/2014) participant identification and a survey of UK stroke physicians. Seven hundred and thirteen ICH patients were admitted during the study period. Sixty nine of these patients were VKA-ICH. Patients not admitted on the acute stroke pathway ( $\mathrm{n}=8)$ or who had palliative care commenced immediately on admission $(n=6)$ were excluded, leaving 55 patients in the final analysis. During $2011 / 12$ we identified and implemented service changes to reduce delays in PCC administration. The primary outcome was the time interval between diagnostic brain scan and commencement of PCC treatment (scan-to-needle time).
\end{abstract}

Secondary outcomes were the time interval between admission and commencement of PCC (door-to-needle time) and symptom onset and commencement of PCC (onset-to-needle time). Three key barriers were identified to rapid administration of PCC, including haematology consultation, collection of PCC from the transfusion laboratory, and obtaining the laboratory INR result. Our survey indicated that these barriers existed at most UK centres. We implemented point-of-care INR testing, moved PCC to the emergency department, and agreed a protocol to administer PCC autonomously. Our scan-to-needle time more than halved, from a median of 127 min (interquartile range (IQR), 111 to $208 \mathrm{~min}$ ) prior to service changes to $58 \mathrm{~min}$ (IQR 50 to $91 \mathrm{~min}$; $\mathrm{p}<0.001$ ) afterwards. We have substantially reduced delays in delivering PCC to VKA-ICH patients at our centre and our simple changes could be easily implemented at centres facing similar barriers.

\section{Problem}

In patients with acute intracerebral haemorrhage $(\mathrm{ICH})$ taking vitamin $\mathrm{K}$ antagonists (VKA; eg warfarin), rapid administration of prothrombin complex concentrate (PCC), and vitamin $\mathrm{K}$ is associated with a reduction in further haematoma expansion and may improve survival and functional outcomes. Given the focus on administering intravenous thrombolysis rapidly to acute ischaemic stroke patients, stroke services in the UK should be well placed to administer PCC to ICH patients in a similar time frame. However, we noted that 'door-to-needle' times (DNT) at our centre were much greater for delivering PCC than for delivering intravenous thrombolysis.

We thus sought to identify barriers to the rapid administration of PCC at our centre and to determine whether these barriers were widespread in UK practice. Prior to any service changes (1/1/2008 to $1 / 12 / 2010$ ), the key processes in reversing anticoagulation following diagnosis of $\mathrm{ICH}$ (figure 1) were laboratory measurement of the international normalised ratio (INR), discussion of each case with the on-call haematologist for approval to use PCC, ordering PCC from blood transfusion, presenting the order form and PCC prescription to the blood transfusion laboratory (a five minute walk from the emergency department (ED)), collection and return of $\mathrm{PCC}$, and reconstitution and administration of PCC to the patient in the ED.

\section{Background}

The incidence of $\mathrm{ICH}$ in patients taking oral anticoagulants is rising, partly due to their increased use for the prevention of ischaemic stroke in patients with atrial fibrillation.[1] Anticoagulant-associated $\mathrm{ICH}$ carries a particularly poor prognosis, with a case-fatality of up to $50 \%$ at three months.[2] Clinically significant haematoma expansion affects a third of all ICH patients and the risk is doubled by anticoagulants.[3] In patients taking a VKA, intravenous replacement of clotting factors and administration of vitamin $\mathrm{K}$ rapidly normalise the INR and may reduce haematoma expansion and improve survival.[4-7] PCC achieves this quicker than fresh frozen plasma,[8] so is recommended as first line treatment in the UK.[9]

Given the high risk of haematoma expansion in the first few hours after onset in $\mathrm{ICH}$ patients on VKAs (VKA-ICH), it is imperative that $P C C$ is given without delay to maximise therapeutic effect. A large observational study of 853 VKA-ICH patients receiving PCC at 19 German centres has been recently published, demonstrating that treatment within four hours of admission to hospital is associated with a significant reduction in haematoma enlargement, when compared to more delayed treatment (19.8\% vs. $41.5 \%$ ). [7] This confirms the rationale for our focus on speed of administration.

\section{Baseline measurement}


BMJ Quality Improvement Reports

In December 2010, we retrospectively identified all vitamin K antagonists (VKA-ICH) cases who presented to our centre via the acute stroke pathway from $1 / 1 / 2008$ to $1 / 12 / 2010$ and who were not immediately palliated in the emergency department (ED). VKA-ICH cases were identified by reviewing the electronic patient records (EPR) of all patients admitted to our hospital with a diagnosis of $\mathrm{ICH}$. ICH cases were initially identified via the hospital coding department from $1 / 1 / 2008$ to $30 / 4 / 2010$ and from $1 / 5 / 2010$ to $31 / 7 / 2014$ via data collected for the Stroke Implementation National Audit Programme (SINAP) and subsequently the Sentinel Stroke National Audit Programme (SSNAP). Survival data were obtained from EPR. We collected clinical information, timings, laboratory results, imaging data (including haematoma volume by the $A B C / 2$ method[10]) from EPR, radiology databases, and transfusion records. We defined the DNT for PCC as the time between arrival in hospital and commencement of PCC infusion.

All patients given PCC received Beriplex (CSL Behring UK Limited). Dosing was $20 \mathrm{IU} / \mathrm{kg}$ if the INR was $\leq 2.5$ and $30 \mathrm{IU} / \mathrm{kg}$ if the INR was $>2.5$, as dictated by our hospital dosing protocol introduced by the haematology department from 9/10/2008. All patients given PCC also received intravenous vitamin $\mathrm{K}(10 \mathrm{mg})$. Upon completion of our initial retrospective analysis, we invited all members of the British Association of Stroke Physicians (BASP) $(n=600)$ to participate in an online survey on 16/12/2010. All data are expressed as median and interquartile range. For the purposes of our analysis, patients were classified according to whether they were admitted before ('pre'), during, or after ('post') implementation of the key service changes. Timings for patients before, during, and after service changes were compared using the Kruskal-Wallis test. All analyses were undertaken using SPSS (version 20, IBM Corp).

One hundred and ninety four $\mathrm{ICH}$ patients were admitted to our centre from $1 / 1 / 2008$ to $1 / 12 / 2010$ including 15 with VKA-ICH (table). Two were treated outwith the acute stroke pathway, leaving 13 cases included in our initial retrospective analysis. One patient did not receive PCC as the physician responsible felt it was not indicated. Three key delays were identified. First, the time from completion of investigations (computed tomography brain scan and INR) to ordering PCC from the transfusion lab was considerable (68 $\mathrm{min} ; 44$ to $81 \mathrm{~min}$ ). Contacting and obtaining approval from the oncall haematologist was considered to be a major component of this delay. Second, an INR result is required to determine whether PCC should be given (indicated if INR $\geq 1.3$ ). We found a median delay of $39 \mathrm{~min}$ (29 to $60 \mathrm{~min}$ ) between admission and the registration of the INR on the lab computer system. Third, we found a delay of 18 $\mathrm{min}$ (14 to $31 \mathrm{~min}$ ) from ordering to collecting PCC, and $43 \mathrm{~min}$ (15 to $63 \mathrm{~min}$ ) from ordering to the start of PCC infusion.

We received 139 responses to our survey (139/600 invited BASP members; $23 \%$ response rate) with responses from all regions in the UK. The majority of respondents indicated that haematology approval, the need to wait for a lab INR result, and storage of PCC away from the point-of-care were barriers at their centre. $76 \%$ reported that they must contact a haematologist to obtain PCC and $67 \%$ had to obtain the PCC from the haematology or transfusion lab. Only $5 \%$ had PCC available where they treat acute stroke patients. A point-of-care INR device was available to only $4 \%$ of respondents.

See supplementary file: ds5202.pdf - "Table and figures 1\&2"

\section{Design}

We collected times for key processes in delivering PCC, identified barriers to rapid treatment, and implemented service changes between 25/1/2011 and 30/12/2012. VKA-ICH cases were prospectively identified from $1 / 12 / 2010$ to $31 / 7 / 2014$ to determine the impact of service changes. Three key delays were identified at our centre (Figure 1), including delay in obtaining an INR result, delays obtaining PCC from haematology, and delays seeking permission for the use of PCC from a haematologist. We thus engaged with haematology and blood transfusion to remove these barriers, by agreeing a shared protocol for the use of PCC in ICH and by moving a stock of PCC to the ED. We approached the Greater Manchester \& Cheshire Cardiac \& Stroke Network who agreed to fund provision of a point-of-care INR testing device to provide a rapid result to inform PCC administration and dosing.

\section{Strategy}

From 25/1/2011, stroke physicians were authorised to request and prescribe PCC without approval from a haematologist, provided treatment was concordant with a local protocol that was developed in collaboration with haematology. Once funding and approvals were obtained, we introduced a hand-held point-of-care INR testing device (Coagucheck XS, Roche Diagnostics Limited) to the ED on 22/12/2011, which measures the INR in less than one minute. Finally, we obtained approval to introduce a stock of PCC in the ED from 30/12/2012.

\section{Results}

Between $25 / 1 / 2011$ to $31 / 7 / 2014,519 \mathrm{ICH}$ cases were admitted including 54 with VKA-ICH. Of VKA-ICH cases, 6 were palliated on admission and 6 were outwith the acute stroke pathway, leaving 42 patients (19 during change implementation, 23 after) included in our final analysis. We found a marked reduction in our scan-to-needle time (Figure 2B) from $127 \mathrm{~min}$ (111 to $208 \mathrm{~min} ; \mathrm{n=12}$ ) prior to service changes to $58 \mathrm{~min}$ ( 50 to $91 \mathrm{~min} ; n=23 ; p<0.001$ ) after changes were implemented, with a concomitant reduction in door-toneedle time (DNT; $218 \mathrm{~min}$ (141 to $289 \mathrm{~min}$ ) 'pre' vs. $108 \mathrm{~min}$ (81 to $136 \mathrm{~min})$ 'post'; $p<0.001)$. Onset-to-needle time improved but this was not statistically significant ((308 $\mathrm{min}$ (243 to $770 \mathrm{~min})$ 'pre' vs. $230 \mathrm{~min}$ (200 to $315 \mathrm{~min}$ ) 'post'; $\mathrm{p}=0.13$ ). The improvement in DNT was partly accounted for by improvement in door-to-scan time of 18 $\min$ (48 min (27 to $75 \mathrm{~min}$ ) 'pre' vs. $30 \mathrm{~min}$ ( 25 to 49 ) 'post'; $p=0.23$ ). Improvement began during change implementation and was sustained for the 1.5 years afterwards.

\section{Lessons and limitations}

The administration of PCC to VKA-ICH patients represents an opportunity to give a treatment to improve the outcome for patients 
with an otherwise poor prognosis. Since completing our study, a large observational study has shown that treatment within four hours of admission to hospital is associated with a significant reduction in haematoma enlargement, when compared to more delayed treatment.[7] This confirms the rationale for our service changes, demonstrating that administering PCC within four hours of admission reduces the risk of haematoma expansion. By implementing point-of-care INR testing, developing a protocol to remove the need for haematology consultation and storage of PCC in the ED, we have more than halved the time taken to treat VKAICH patients at our centre. Prior to our service changes, 7/13 (54\%) patients received PCC within four hours. During the period of service change this improved to $16 / 19$ (84\%). Following service changes, treatment within four hours was achieved for every patient $(23 / 23 ; 100 \%)$. Given that treatment within four hours is associated with a reduction in haematoma growth, this improvement in the speed of delivery of PCC may translate to improved patient outcomes.

It is important to note that in April 2010 our centre became a regional comprehensive stroke centre, accepting patients directly from a catchment population of 2.68 million. The stroke service at our hospital had previously accepted patients from a considerably smaller catchment of around 250,000. This step change in working patterns and patient numbers is very likely to have impacted upon processes of care, but only 6 of the 13 patients included in the 'prechange' group were admitted prior to service reconfiguration. Nevertheless, the clear improvement seen in door-to-scan time is likely to have been driven by the change to comprehensive stroke centre status and would be expected to partly account for the improvement in door-to-needle time. Onset-to-door times also showed a small, non-significant $(p=0.83)$ improvement from 149 min (67 to $853 \mathrm{~min}$ ) 'pre' to $116 \mathrm{~min}$ (68 to $199 \mathrm{~min}$ ) 'post', which will partly account for the non-significant improvement in onset-toneedle times. It is likely that the change in onset-to-needle time did not reach statistical significance due to the marked variation of onset-to-door times during the period prior to service change implementation. However, our primary finding of a marked and significant improvement in scan-to-needle time would be expected to be achieved largely by improvements in processes specific to delivery of PCC, rather than the general impact of change to comprehensive stroke centre status.

\section{Conclusion}

To further cut delays in giving PCC, we plan to implement prospective review of all VKA-ICH cases at stroke team meetings to improve staff awareness and identify and address any delays as they emerge. Awareness of the protocol for VKA-ICH treatment will also be incorporated in new staff induction. Although we have largely focused on DNT for our centre it is important to note that other factors may influence the efficacy of this treatment. Different preparations of PCC are available and vary from country to country, divided in to three or four factor PCCs, and there is evidence to suggest that four factor PCC is superior to three factor PCC in correcting the INR.[11] Also, it is important to ensure that an adequate dose is administered, as determined by the patient's weight and their admission INR.[12] Newer anticoagulants have recently been licensed (e.g. rivaroxaban, dabigatran, apixaban) and although there is uncertainty about how to manage life-threatening bleeding with these agents,[11] it will still be necessary to attempt to normalise clotting as quickly as possible.

\section{References}

1. Smith NL, Psaty BM, Furberg CD, et al. Temporal trends in the use of anticoagulants among older adults with atrial fibrillation. Arch Intern Med 1999;159:1574-8.

2. Meretoja A, Strbian D, Putaala J, et al. SMASH-U: A Proposal for Etiologic Classification of Intracerebral Hemorrhage. Stroke 2012;43:2592-7. doi:10.1161/STROKEAHA.112.661603.

3. Brouwers HB, Chang Y, Falcone GJ, et al. Predicting hematoma expansion after primary intracerebral hemorrhage. JAMA Neurol 2014;71:158-64. doi:10.1001/jamaneurol.2013.5433.

4. Huttner HB, Schellinger PD, Hartmann M, et al. Hematoma Growth and Outcome in Treated Neurocritical Care Patients With Intracerebral Hemorrhage Related to Oral Anticoagulant Therapy Comparison of Acute Treatment Strategies Using Vitamin K, Fresh Frozen Plasma, and Prothrombin Complex Concentrates. Stroke 2006;37:1465-70. doi:10.1161/01.STR.0000221786.81354.d6.

5. Huhtakangas J, Tetri S, Juvela S, et al. Improved survival of patients with warfarin-associated intracerebral haemorrhage: a retrospective longitudinal population-based study. Int J Stroke Published Online First: 11 December 2012. doi:10.1111/j.1747-4949.2012.00926.x.

6. Hanger HC, Geddes JAA, Wilkinson TJ, et al. Warfarinrelated intracerebral haemorrhage: better outcomes when reversal includes prothrombin complex concentrates. Intern Med J 2013;43:308-16. doi:10.1111/imj.12034.

7. Kuramatsu JB, Gerner ST, Schellinger PD, et al. Anticoagulant reversal, blood pressure levels, and anticoagulant resumption in patients with anticoagulationrelated intracerebral hemorrhage. JAMA 2015;313:824-36. doi:10.1001/jama.2015.0846.

8. Hickey M, Gatien M, Taljaard M, et al. Outcomes of urgent warfarin reversal with frozen plasma versus prothrombin complex concentrate in the emergency department.

Circulation 2013;128:360-4. doi:10.1161/CIRCULATIONAHA.113.001875.

9. Intercollegiate Stroke Working Party. National clinical guideline for stroke, 4th edition. London: Royal College of Physicians 2012.

10. Kothari RU, Brott T, Broderick JP, et al. The ABCs of Measuring Intracerebral Hemorrhage Volumes. Stroke 1996;27:1304-5.

11. Dzik WS. Reversal of drug-induced anticoagulation: old solutions and new problems. Transfusion 2012;52 Suppl 1:45S - 55S. doi:10.1111/j.1537-2995.2012.03690.x.

12. Masotti L, Di Napoli M, Godoy DA, et al. The practical management of intracerebral hemorrhage associated with oral anticoagulant therapy. Int J Stroke 2011;6:228-40. doi:10.1111/j.1747-4949.2011.00595.x 


\section{Declaration of interests}

Competing Interests: None.

\section{Acknowledgements}

Lydia Baxter, Edith Wood, Martin Thomas, Andy Vail, Charles

Sherrington, Pippa Tyrrell.

\section{Ethical approval}

Ethics committee approval: This work was done as part of a larger service development and research project which received NHS REC approval (11/H1011/3) and R\&D from Salford R\&D (2010/337 NEURO). 